\title{
Regulators hidden in human proteome
}

A search for DNA-protein interactions has unexpectedly pulled out over 300 human proteins that are known for other biological roles. By highlighting the multitasking function of many human proteins, this work provides a new perspective on the molecular basis of organismal complexity.

The study used protein microarrays to examine the DNA binding specificities of almost 4,200 recombinant proteins. In the protein mix the authors included not only known transcription factors (TFs) - of

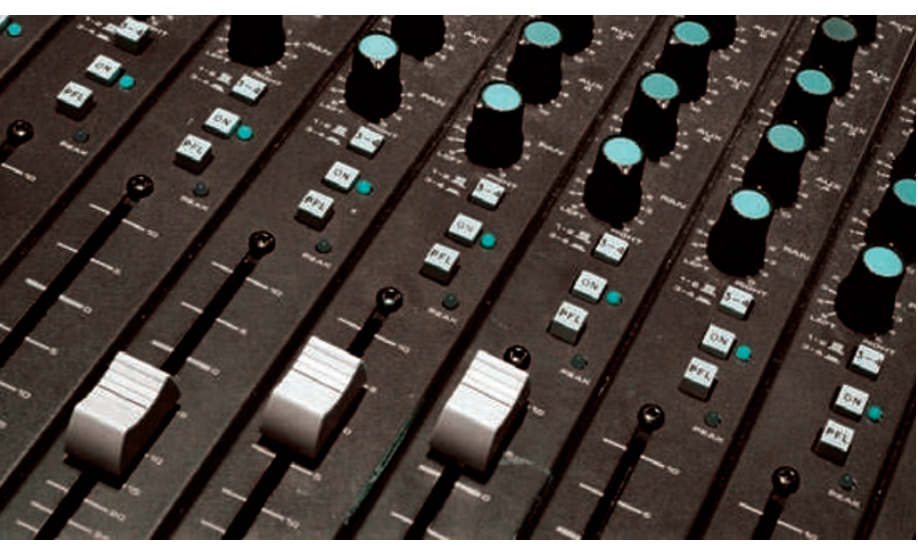

which there are predicted to be up to 1,700 in the human genome - but also proteins that are not thought to directly contact DNA, such as signalling molecules and RNAbinding proteins. The proteins were then tested individually for their ability to bind 460 DNA sequences of 6-34 bp that were predicted to regulate transcription.

The assay identified the binding specificity of many proteins that are already known to be TFs and, surprisingly, also revealed 367 nonTF proteins that could directly bind DNA. This last category included extracellular signal-regulated kinase 2 (ERK2, also known as mitogen-activated protein kinase 1), a conserved and extensively studied molecule with functions in developmental patterning.

The authors went on to explore the significance of ERK2-DNA interaction. In vitro, ERK2 binds a consensus DNA motif and can repress a downstream reporter gene - a function that is independent of the kinase activity of ERK2. When ERK2 was knocked down in HeLa cells by small interfering RNA, 82 genes were upregulated at least 2 -fold. In 11 of the 21 putative target genes tested, ERK2 was indeed associated with its DNArecognition sequence. In particular, the authors describe how ERK2 represses the expression of genes (OAS1 and IRF9) that are induced by interferon- $\gamma$ by competitively binding to motifs in the promoters of these genes.

The possibility that the complexity of some organisms derives from the undiscovered transcriptional regulatory activity of many proteins that are not thought to be TFs adds a new layer to genotype-phenotype relationships. This study has doubled the number of known DNA-protein interactions in humans, but given that the authors looked at only $20 \%$ of proteins, the full extent of this complexity could be much higher.

Tanita Casci

ORIGINAL RESEARCH PAPER Hu, S., Xie, Z. et al. Profiling the human protein-DNA interactome reveals ERK2 as a transcriptional repressor of interferon signaling. Cell 139, 610-622 (2009) 